LPTHE-P04-04

hep-ph/0407368

\title{
FALL AND RISE OF THE GLUON SPLITTING FUNCTION*
}

\author{
Gavin P. Salam \\ LPTHE, Universities of Paris VI \& VII and CNRS, \\ 75252 Paris 75005, France.
}

\begin{abstract}
This talk reviews some recent results on the NLL resummed small$x$ gluon splitting function, as determined including renormalisation-group improvements. It also discusses the observation that the LO, NLO, NNLO, etc. hierarchy for the gluon splitting function breaks down not when $\alpha_{s} \ln 1 / x \sim 1$ but rather for $\alpha_{s} \ln ^{2} 1 / x \sim 1$.
\end{abstract}

It is well-known that the resummation of leading-logarithms of $x$ (LLx) [1] and next-to-leading logarithms (NLL $x)[2,3]$ in the gluon splitting function,

$x P_{g g}(x)=\sum_{n=1} A_{n, n-1} \bar{\alpha}_{s}^{n} \ln ^{n-1} \frac{1}{x}+\sum_{n=2} A_{n, n-2} \bar{\alpha}_{s}^{n} \ln ^{n-2} \frac{1}{x}+\ldots, \quad \bar{\alpha}_{s}=\frac{\alpha_{s} N_{c}}{\pi}$,

leads to strong small- $x$ growth (positive at LL $x$, negative at NLL $x$ ), quite inconsistent with data $[4,5]$. In contrast, fits to data with purely NLO splitting functions, free of any small- $x$ enhancements (because $A_{21}=0$ ), work remarkably well $[6,7]$ even at $x \sim 10^{-4}$, where one would expect the effects of resummation to be relevant.

This apparent paradox, and related issues, have been the subject of intense investigation over the past few years [8-19], and have become all the more relevant with the recent calculation of the NNLO splitting functions [20], which explicitly show the first of the small- $x$ enhanced terms, $A_{31}$ (the LL $x A_{32}$ term is also zero).

A characteristic of nearly all such approaches is that the resummed $P_{g g}$ splitting function ends up bearing a strong similarity to the NLO result, the main additional features being a dip in the splitting function at moderately small values of $x \sim 10^{-3}$, followed by a slow rise at very small $x$.

The slowness of the rise has been understood in $[10,12,15]$ as being, in part, a consequence of running coupling effects, which convert the branch cut of the fixed-coupling LL $x$ splitting function into a set of poles with weak, $\mathcal{O}\left(\alpha_{s}^{2}\right)$, residues. Furthermore the position of the dominant pole corresponds to a lower small- $x$ power rise than the position of the corresponding fixedcoupling branch point, the difference in the power being of order $b^{2 / 3} \alpha_{s}^{5 / 3}$, where

\footnotetext{
*Talk presented at DIS 2004, Strbské Pleso, Slovakia, April 2004, and at the Eighth Workshop on Non-Perturbative Quantum Chromodynamics, Paris, France, June 2004.
} 
$b=\left(11-\frac{2 n_{f}}{3}\right) / 12$. Just as important, is the consistent all-orders treatment of renormalisation group logarithms in the BFKL evolution kernel [13-15].

The dip, instead, had until recently received less attention, even though it appeared in all approaches, and is likely, at today's energies, to be phenomenologically more relevant than the asymptotically small- $x$ rise.

Before discussing the dip in detail, it is useful review the methods, as used in [17], to extract the resummed small- $x$ splitting function that will be shown here. First one introduces a kernel $\mathcal{K}\left(z, k, k^{\prime}\right)$ which includes both BFKL (LL $x$, NLL $x$ ) and renormalisation group (RG, i.e. LO DGLAP) contributions to small$x$ evolution. It is designed so as to be symmetric under the exchange of the two transverse scales, $k_{0}$ and $k_{1}$, modulo symmetry-breaking running-coupling effects. One then numerically determines a gluon Green function $G\left(\ln 1 / x, k, k_{0}\right)$, as the solution of

$$
G\left(\ln 1 / x, k, k_{0}\right)=G_{0}\left(\ln 1 / x, k, k_{0}\right)+\mathcal{K} \otimes G,
$$

where the inhomogeneous term $\left(G_{0}\right)$ represents some arbitrary initial condition and the convolution $\mathcal{K} \otimes G$ is over both longitudinal momenta $x$ and transverse momenta $k$. The Green function can, among other things, be used as an input for studies of processes such as $\gamma^{*} \gamma^{*}$, in which case one takes $k \sim k_{0} \gg \Lambda_{Q C D}$. This is illustrated in fig $\square$ where the curves labelled 'scheme A' and ' $\mathrm{B}$ ' correspond to two variants of the RG-improved NLL $x$ kernel. Features to note include the slow onset of the BFKL growth of the Green function compared to LL $x$ evolution, and the good degree of stability of the RG improved approach compared to pure NLL $x$ approaches, the latter being sensitive both to the details of the treatment of the running coupling as well as to the precise definition of $Y\left(\ln s / k k_{0}\right.$ or $\ln s / k^{2}$, not shown) ${ }^{1}$

Rather than such same-scale processes, here our main interest is in cases where $k_{0}$ is some non-perturbative scale $\sim \Lambda_{Q C D}$, while $k \gg k_{0}$. One can then introduce a gluon distribution,

$$
x g\left(x, Q^{2}\right) \equiv \int^{Q} d^{2} k G^{\left(\nu_{0}=k^{2}\right)}\left(\ln 1 / x, k, k_{0}\right), \quad x=\frac{k^{2}}{s} .
$$

Perturbatively, $g\left(x, Q^{2}\right)$ is not under control, since it depends on non-perturbative information such as the initial condition $G^{(0)}$, as well as the detailed dynamics in the infrared. However, because collinear factorisation holds even at small- $x$, the splitting function $P_{g g}\left(z, Q^{2}\right)$ is perturbatively defined, through the following relation,

$$
\frac{d g\left(x, Q^{2}\right)}{d \ln Q^{2}}=\int \frac{d z}{z} P_{g g}\left(z, Q^{2}\right) g\left(\frac{x}{z}, Q^{2}\right) .
$$

Thus one can numerically evaluate the gluon distribution for a particular set of non-perturbative criteria (e.g. infrared cutoff on $\alpha_{s}$ ) and then carry out the

\footnotetext{
${ }^{1}$ Another running coupling scheme for pure NLL $x$ has also been considered in [21], giving results similar to our $\alpha_{s}\left(q^{2}\right)$ curves. It is within an approach that has the advantage of providing easy access to information also on the dependence of $G\left(\ln 1 / x, \vec{k}, \vec{k}_{0}\right)$ on the azimuthal angle between $\vec{k}$ and $\vec{k}_{0}$
} 

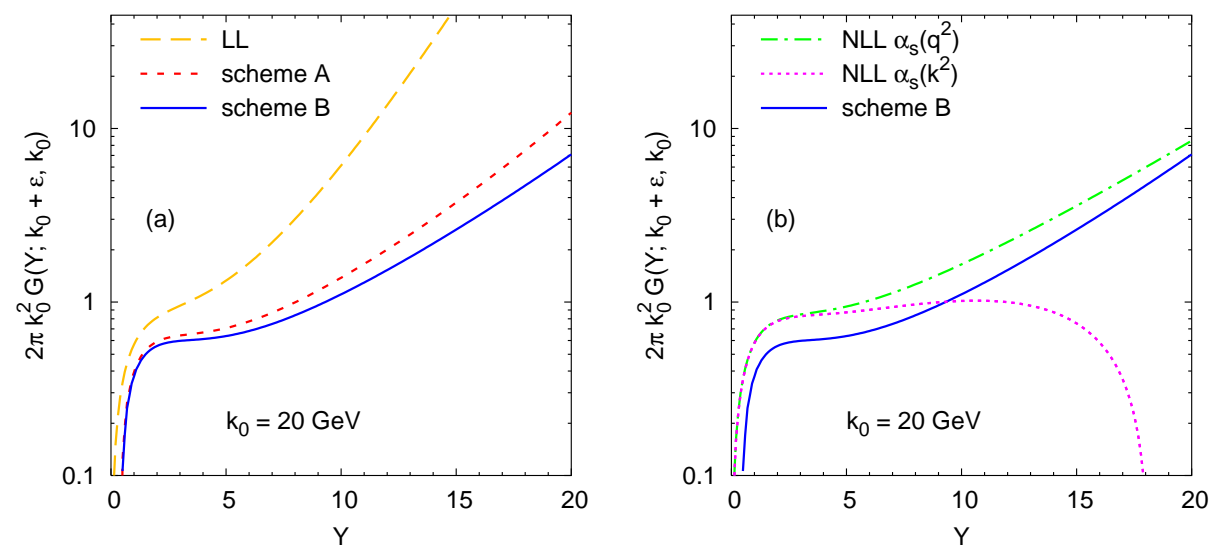

Figure 1: Gluon Green function, as relevant to problems such as $\gamma^{*} \gamma^{*}$ scattering, with two hard scales, $k$ and $k_{0}$, and $Y \equiv \ln s / k k_{0}$. Left: compared to LL $x$ BFKL evolution. Schemes A and B represent two forms of RG improvement of the NLL $x$ BFKL kernel. Right: comparison to pure NLL $x$ evolution with two different schemes for the running of the coupling [17].

deconvolution so as to obtain $P_{g g}$. This gives the splitting function of fig 2 $\left(\mathrm{NLL}_{\mathrm{B}}\right.$, corresponding to scheme B of fig @), together with an inner uncertainty band, associated with an estimate of residual (higher-twist) dependence on the non-perturbative dynamics. The small size of the latter serves as a cross-check of factorisation.

Relevant features of the splitting function of fig 2 include the dip at moderately small $x$, followed by the slow rise at much smaller $x$. To within renormalisation scale uncertainties (outer shaded band) it is compatible with the inverse Mellin transform of the anomalous dimension determined in [15], which was based on similar physical assumptions ( $\omega$-expansion) but with different subleading terms, thus confirming the overall consistency between different approaches. Regarding the potential for future comparisons with data, an interesting similarity was observed in [11] with the splitting function of [8], which had in part been fitted to the HERA data.

As already mentioned, the main feature of the splitting function in the phenomenologically relevant region of $x \sim 10^{-3}$ is a dip. It is important that one understand the origin of the dip, and establish whether it is a robust small- $x$ prediction or rather some form of artefact.

A first point of interest in this respect concerns the striking similarity, down to $x \sim 10^{-3}$, with the recently calculated NNLO splitting function [20]. Since the LL $x A_{32}$ term is zero, the NNLO splitting function, at small- $x$, is dominated by a (NLL $x)$ term, $A_{31} \bar{\alpha}_{s}^{3} \ln 1 / x$, where $A_{31}$ is negative. It also contains a NNLL $x$ term, $A_{30} \bar{\alpha}_{s}^{3}$, but this contributes only an overall shift to the height of the curve, without modifying its $x$-dependence. This suggests that for moderately small $x$ it might be the $A_{31} \bar{\alpha}_{s}^{3} \ln 1 / x$ term that dominates the resummed 


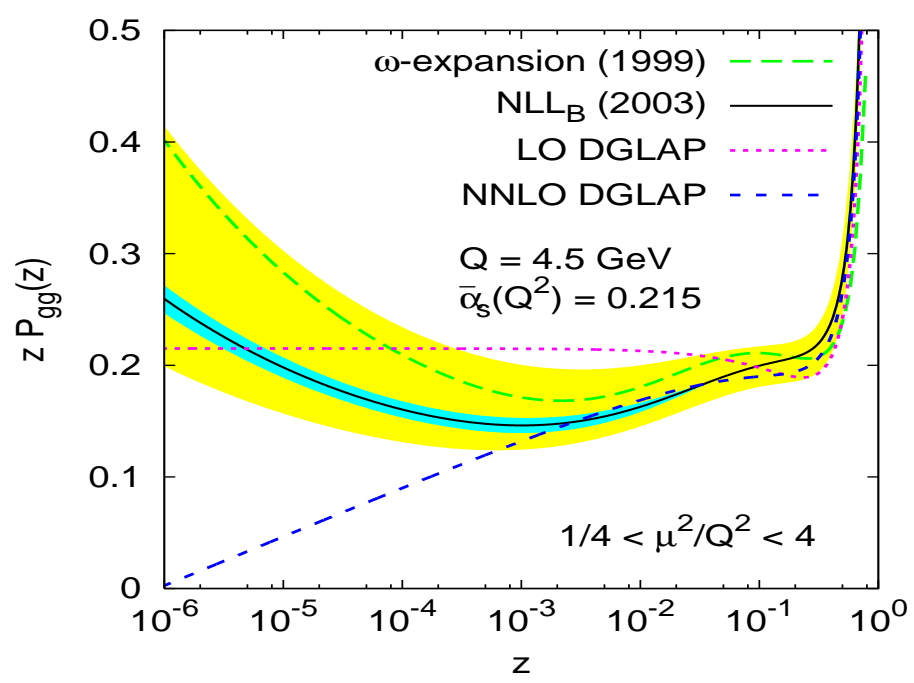

Figure 2: $P_{g g}$ resummed small- $x$ splitting function, as determined in [17], together with a shaded band from the variation of the renormalisation scale in the resummation. It is compared to an older resummed determination, in the $\omega$ expansion [15], the LO DGLAP splitting function and the recently determined NNLO splitting function [20].

splitting function. To see whether this is the case, one has to examine the relative size of other logarithmically enhanced terms. At the next order in $\alpha_{s}$, the term with the strongest $x$ dependence is $A_{43} \bar{\alpha}_{s}^{4} \ln ^{3} 1 / x$. Taking just the $A_{31}$ and $A_{43}$ terms gives (ignoring constant terms of order $\alpha_{s}^{2}$ ),

$$
x P_{g g}(x) \simeq \alpha_{s}+A_{31} \bar{\alpha}_{s}^{3} \ln 1 / x+A_{43} \bar{\alpha}_{s}^{4} \ln ^{3} 1 / x,
$$

which has a minimum at $\ln 1 / x=\sqrt{-\frac{A_{31}}{3 A_{43}} \frac{1}{\bar{\alpha}_{s}}}$, since $A_{31}$ and $A_{43}$ have opposite signs. The appearance here of $1 / \sqrt{\bar{\alpha}_{s}}$ is quite unexpected, since small- $x$ resummation effects are normally expected to set in for $\bar{\alpha}_{s} \ln 1 / x \sim 1$. It is a consequence of the absence of the $A_{21} \bar{\alpha}_{s}^{2} \ln 1 / x$ and $A_{32} \bar{\alpha}_{s}^{3} \ln ^{2} 1 / x$ LL $x$ terms.

Having established the existence of two terms leading to a dip structure for $\alpha_{s} \ln ^{2} x \sim 1$, the next step is to examine the impact of yet higher-order terms. Fig 3 illustrates the various terms that are present, highlighting the classification in the normal LLx hierarchy, the number of powers of $\alpha_{s}$ and of $\ln x$. One sees that eq. (15), i.e. the terms grouped in the upper (red) ellipse, includes the complete set of terms of order $\alpha_{s}^{5 / 2}$ for $\alpha_{s} \ln ^{2} x \sim 1$. One can also identify a finite set of terms (lower, blue ellipse) that contribute at order $\alpha_{s}^{3}$ in this region and so forth. This ability to classify all contributions into finite groups of terms of a common order of magnitude for $\alpha_{s} \ln ^{2} x \sim 1$ means that our identification of a dip associated with the structure of eq.(15) is robust. We 

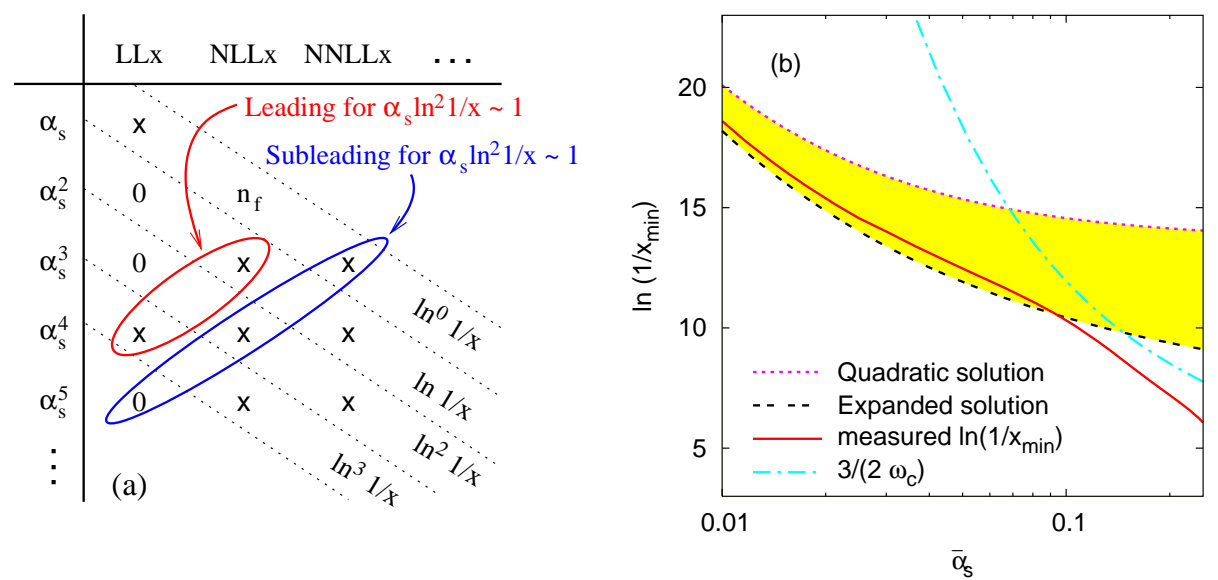

Figure 3: (a) Various classifications of small- $x$ logarithmically enhanced terms, $n_{f}$ indicating that a term is proportional to $n_{f}$. (b) The position of the dip as a function of $\bar{\alpha}_{s}$, as measured from the numerical calculations of [17]; as estimated from the expanded solution, eq.(6), together with an uncertainty band (see [22] for details); and compared to $3 /\left(2 \omega_{c}\right)$, an upper bound for $\ln 1 / x_{m i n}$, based on resummation arguments.

can write an expansion for its position [22],

$$
\ln \frac{1}{x_{\min }}=\sqrt{-\frac{A_{31}}{3 A_{43}} \frac{1}{\bar{\alpha}_{s}}}-\frac{A_{42}}{3 A_{43}}+\mathcal{O}\left(\sqrt{\bar{\alpha}_{s}}\right) \simeq \frac{1.156}{\sqrt{\bar{\alpha}_{s}}}+6.947+\mathcal{O}\left(\sqrt{\bar{\alpha}_{s}}\right),
$$

while its depth $-d$ below the level of the constant $\bar{\alpha}_{s}$ and $\bar{\alpha}_{s}^{2}$ contributions is

$$
\begin{aligned}
-d & =\frac{2 A_{31}}{9} \sqrt{\frac{-3 A_{31}}{A_{43}}} \bar{\alpha}_{s}^{5 / 2}-\frac{1}{3} \frac{A_{31} A_{42}}{A_{43}} \bar{\alpha}_{s}^{3}+\mathcal{O}\left(\bar{\alpha}_{s}^{7 / 2}\right) \\
& \simeq-1.237 \bar{\alpha}_{s}^{5 / 2}-11.15 \bar{\alpha}_{s}^{3}+\mathcal{O}\left(\bar{\alpha}_{s}^{7 / 2}\right) .
\end{aligned}
$$

Given that the expansion is in powers of $\sqrt{\alpha_{s}}$, the large coefficients of the higherorder terms (given for $n_{f}=4$ ) mean that one can only trust the expansion for extremely small values of $\alpha_{s}$, and one may legitimately wonder whether the discussion given here is of relevance to the dip seen for realistic values of $\alpha_{s}$.

To help answer this question, fig $3 \mathrm{p}$ shows the position of the dip as measured from the numerical calculations of [17] (solid line) compared to eq. (6) (dashed line) together with a band indicating the estimated size of $\mathcal{O}\left(\sqrt{\alpha_{s}}\right)$ uncertainties on eq. (6). There is remarkably good agreement up to $\bar{\alpha}_{s} \simeq 0.1$, despite the fact that eq.(6) seems to converge only for much smaller $\alpha_{s}$.

Beyond $\bar{\alpha}_{s} \simeq 0.1$ one sees a change in behaviour of the position of the minimum. To understand it, we note that the usual (fixed-coupling) approximation for the resummed splitting function is

$$
x P_{g g}(x) \sim \frac{x^{-\omega_{c}}}{\ln ^{3 / 2} 1 / x},
$$


where $\omega_{c}$ is the asymptotic power for the growth of $x P_{g g}(x)$. The point where eq. (8) starts to rise, $\ln 1 / x \sim 3 /\left(2 \omega_{c}\right)$, corresponds to that where the all-orders resummation of $\alpha_{s} \ln 1 / x$ terms becomes important. This point would be expected to provide an upper bound on the position of any minimum of $x P_{g g}(x)$, and indeed, plotting it in fig $3 \mathrm{~b}$ reveals that the kink in the $\bar{\alpha}_{s}$-dependence of $\ln 1 / x_{\min }$ occurs precisely at the point where the $\ln 1 / x \sim 3 /\left(2 \omega_{c}\right)$ bound becomes relevant. What occurs here is that the upper bound, of order $1 / \alpha_{s}$, becomes smaller than the formal position of the minimum, of order $1 / \sqrt{\alpha_{s}}$, as a consequence of the relative sizes of the (subleading) coefficients of the two series.

Overall, we arrive at the following overall picture regarding the dip: the initial decrease seems in all cases, including for realistic values of $\alpha_{s}$, to be due to the negative $A_{31} \bar{\alpha}_{s}^{3} \ln 1 / x$ term. For small $\alpha_{s}$, the decrease continues up to the point, $\ln 1 / x \sim 1 / \sqrt{\alpha_{s}}$, where the $A_{43} \bar{\alpha}_{s}^{4} \ln ^{3} 1 / x$ becomes of similar order, cf. eq.(6). But for realistic values of $\alpha_{s}$, the full BFKL rise actually sets in earlier than is given by eq. (66), roughly for $\ln 1 / x \sim 3 /\left(2 \omega_{c}\right)$ and it is this point that then determines the position of minimum of the dip. This global picture leads us to believe that the dip is a rigorous property of the small- $x$ expansion, even if it is difficult to calculate its properties analytically for phenomenologically relevant values of $\alpha_{s}$.

Acknowledgements. The work described here has been carried out in collaboration with M. Ciafaloni, D. Colferai and A. Staśto.

\section{References}

[1] L.N. Lipatov, Sov. J. Nucl. Phys. 23 (1976) 338; E.A. Kuraev, L.N. Lipatov and V.S. Fadin, Sov. Phys. JETP 45 (1977) 199; I.I. Balitsky and L.N. Lipatov, Sov. J. Nucl. Phys. 28 (1978) 822; L.N. Lipatov, Sov. Phys. JETP 63 (1986) 904.

[2] V.S. Fadin and L.N. Lipatov, Phys. Lett. B 429 (1998) 127.

[3] G. Camici and M. Ciafaloni, Phys. Lett. B 412 (1997) 396, [Erratum-ibid. B 417 (1997) 390]; Phys. Lett. B 430 (1998) 349.

[4] R. K. Ellis, F. Hautmann and B. R. Webber, Phys. Lett. B 348 (1995) 582.

[5] R. D. Ball and S. Forte, Phys. Lett. B 351 (1995) 313.

[6] J. Pumplin et al., JHEP 0207 (2002) 012.

[7] A. D. Martin et al., Eur. Phys. J. C 23 (2002) 73.

[8] G. Altarelli, R.D. Ball and S. Forte, Nucl. Phys. B 575 (2000) 313; Nucl. Phys. B 599 (2001) 383.

[9] G. Altarelli, R.D. Ball and S. Forte, Nucl. Phys. B 621 (2002) 359. 
[10] G. Altarelli, R. D. Ball and S. Forte, Nucl. Phys. B 674 (2003) 459.

[11] G. Altarelli, R. D. Ball and S. Forte, hep-ph/0310016.

[12] R.S. Thorne, Phys. Rev. D 64 (2001) 074005; Phys. Lett. B 474 (2000) 372 .

[13] G.P. Salam, JHEP 9807 (1998) 019.

[14] M. Ciafaloni and D. Colferai, Phys. Lett. B 452 (1999) 372.

[15] M. Ciafaloni, D. Colferai and G.P. Salam, Phys. Rev. D 60 (1999) 114036.

[16] M. Ciafaloni, D. Colferai and G. P. Salam, JHEP 0007 (2000) 054.

[17] M. Ciafaloni et al., Phys. Lett. B 576 (2003) 143; Phys. Rev. D 68 (2003) 114003.

[18] C.R. Schmidt, Phys. Rev. D 60 (1999) 074003.

[19] J. R. Forshaw, D. A. Ross and A. Sabio Vera, Phys. Lett. B 455 (1999) 273.

[20] S. Moch, J. A. M. Vermaseren and A. Vogt, Nucl. Phys. B 646 (2002) 181; Nucl. Phys. B 688 (2004) 101; hep-ph/0404111.

[21] J. R. Andersen and A. Sabio Vera, Phys. Lett. B 567 (2003) 116. Nucl. Phys. B 679 (2004) 345.

[22] M. Ciafaloni et al., Phys. Lett. B 587 (2004) 87. 